\title{
When a partial Borel order is linearizable
}

\author{
by
}

\author{
Vladimir Kanovei (Moscow)
}

\begin{abstract}
We prove a classification theorem of the "Glimm-Effros" type for Borel order relations: a Borel partial order on the reals either is Borel linearizable or includes a copy of a certain Borel partial order $\leq_{0}$ which is not Borel linearizable.
\end{abstract}

Notation. A binary relation $\preccurlyeq$ on a set $X$ is a partial quasi-order, or p.q.-o. in brief, on $X$, iff $x \preccurlyeq y \wedge y \preccurlyeq z \Rightarrow x \preccurlyeq z$, and $x \preccurlyeq x$ for any $x \in X$. In this case, $\approx$ is the associated equivalence relation, i.e. $x \approx y$ iff $x \preccurlyeq y \wedge y \preccurlyeq x$.

If in addition $x \approx x \Rightarrow x=x$ for any $x$ then $\preccurlyeq$ is a partial order, or p.o., so that, say, forcing relations are p.q.-o.'s, but, generally speaking, not p.o.'s in this terminology.

A p.o. is linear (1.o.) iff we have $x \preccurlyeq y \vee y \preccurlyeq x$ for all $x, y \in X$.

Let $\preccurlyeq$ and $\preccurlyeq^{\prime}$ be p.q.-o.'s on resp. $X$ and $X^{\prime}$. A map $h: X \rightarrow X^{\prime}$ will be called half order preserving, or h.o.p., iff $x \preccurlyeq y \Rightarrow h(x) \preccurlyeq^{\prime} h(y)$.

Definition 1. A Borel p.q.-o. $\langle X ; \preccurlyeq\rangle$ is Borel linearizable iff there is a Borel l.o. $\left\langle X^{\prime} ; \preccurlyeq^{\prime}\right\rangle$ and a Borel h.o.p. map $h: X \rightarrow X^{\prime}$ (called a linearization map) satisfying $x \approx y \Leftrightarrow h(x)=h(y)\left(^{1}\right)$.

Introduction. Harrington, Marker, and Shelah [2] proved several theorems on Borel partial order relarions, mainly concerning thin p.q.-o.'s, i.e. those which do not admit uncountable pairwise incomparable subsets. In particular, they demonstrated that any such Borel p.q.-o. is Borel linearizable, and moreover the corresponding l.o. $\left\langle X^{\prime} ; \preccurlyeq^{\prime}\right\rangle$ can be chosen as a suborder of $\left\langle 2^{\alpha} ; \leq_{\text {lex }}\right\rangle$ for some $\alpha<\omega_{1}$, where $\leq_{\text {lex }}$ is the lexicographical order.

Key words and phrases: Borel partial order, Borel linear order.

1991 Mathematics Subject Classification: 03E15, 04A15.

This paper was accomplished during my visit to Caltech in April 1997. I thank Caltech for support and A. S. Kechris and J. Zapletal for useful information and interesting discussions relevant to the topic of this paper during the visit.

$\left({ }^{1}\right)$ The equivalence cannot be dropped in this definition as otherwise a one-element set $X^{\prime}$ works in any case. 
As elementary examples show that thinness is not a necessary condition for Borel linearizability, this result leaves open the problem of linearization of non-thin Borel p.q.-o.'s. Harrington et al. wrote in [2] that "there is little to say about nonthin orderings", although there are many interesting among them like the dominance order on $\omega^{\omega}$.

Our main result will say that not all Borel p.q.-o.'s are Borel linearizable, and there exists a minimal one, in a certain sense, among them.

Definition 2. Let $a, b \in 2^{\omega}$. We define $a \leq_{0} b$ iff either $a=b$ or $a \mathrm{E}_{0} b\left({ }^{2}\right)$ and $a\left(k_{0}\right)<b\left(k_{0}\right)$ where $k_{0}$ is the largest $k$ such that $a(k) \neq b(k)\left({ }^{3}\right)$.

The relation $\leq_{0}$ is a Borel p.q.-o. on $2^{\omega}$ which orders every $\mathrm{E}_{0}$-class similarly to the integers $\mathbb{Z}$ (except for the class $[\omega \times\{0\}]_{\mathrm{E}_{0}}$ ordered as $\omega$ and the class $[\omega \times\{1\}]_{E_{0}}$ ordered as $\omega^{*}$, the inverted order) but leaves any two $\mathrm{E}_{0}$-inequivalent reals incomparable.

The following is the main result of the paper.

TheOrem 3. Suppose that $\preccurlyeq$ is a Borel p.q.-o. on $\mathcal{N}=\omega^{\omega}$. Then exactly one of the following two conditions is satisfied:

$(\mathrm{I}) \preccurlyeq$ is Borel linearizable; moreover $\left({ }^{4}\right)$, there exist an ordinal $\alpha<\omega_{1}$ and a Borel linearization map $h:\langle\mathcal{N} ; \preccurlyeq\rangle \rightarrow\left\langle 2^{\alpha} ; \leq_{\text {lex }}\right\rangle$.

(II) there exists a continuous 1-1 map $F: 2^{\omega} \rightarrow \mathcal{N}$ such that we have $a \leq_{0} b \Rightarrow F(a) \preccurlyeq F(b)$ while $a \mathbb{Z}_{0} b$ implies that $F(a)$ and $F(b)$ are $\preccurlyeq$ incomparable $\left({ }^{5}\right)$.

The theorem resembles the case of Borel equivalence relations where a necessary and sufficient condition for a Borel equivalence relation $E$ to be smooth is that $\mathrm{E}_{0}$ (which is not smooth) does not continuously embed in $\mathrm{E}$ (Harrington, Kechris and Louveau [1]). ( $\leq_{0}$ itself is not Borel linearizable.)

The proof is essentially a combination of ideas and techniques in $[1,2]$.

1. Incompatibility. Let us first prove that (I) and (II) are incompatible.

$\left({ }^{2}\right)$ That is, $a(k)=b(k)$ for all but finite $k$, the Vitali equivalence relation on $2^{\omega}$.

$\left({ }^{3}\right)$ If one enlarges $<_{0}$ so that, in addition, $a<_{0} b$ whenever $a, b \in 2^{\omega}$ are such that $a(k)=1$ and $b(k)=0$ for all but finite $k$ then the enlarged relation can be induced by a Borel action of $\mathbb{Z}$ on $2^{\omega}$, such that $a<_{0} b$ iff $a=z b$ for some $z \in \mathbb{Z}, z>0$.

$\left({ }^{4}\right)$ The "moreover" assertion is an immediate corollary of the linearizability by the above-mentioned result of [2].

$\left({ }^{5}\right)$ Then $F$ associates a chain $\left\{F(b): b \mathrm{E}_{0} a\right\}$ in $\langle\mathcal{N} ; \preccurlyeq\rangle$ to each $\mathrm{E}_{0}$-class $[a]_{\mathrm{E}_{0}}$ so that any two different chains do not contain $\preccurlyeq$ comparable elements: let us call them fully incomparable chains. Thus (II) essentially says that $\preccurlyeq$ admits an effectively "large" Borel family of fully incomparable chains, which is therefore necessary and sufficient for $\preccurlyeq$ to be not Borel linearizable. 
Suppose otherwise. The superposition of the maps $F$ and $h$ is then a Borel h.o.p. map $\phi:\left\langle 2^{\omega} ; \leq_{0}\right\rangle \rightarrow\left\langle 2^{\alpha} ; \leq_{\text {lex }}\right\rangle$ satisfying the following: $\phi(a)=\phi(b)$ implies that $a \mathrm{E}_{0} b$, i.e. $a$ and $b$ are $\leq_{0}$ comparable.

Therefore, as any $\mathrm{E}_{0}$-class is $\leq_{0}$-ordered similarly to $\mathbb{Z}, \omega$, or $\omega^{*}$, the $\phi$-image $X_{a}=\phi "[a]_{\mathrm{E}_{0}}$ of the $\mathrm{E}_{0}$-class of any $a \in 2^{\omega}$ is $\leq_{\text {lex }}$-ordered similarly to a subset of $\mathbb{Z}$. If $X_{a}=\left\{x_{a}\right\}$ is a singleton then put $\psi(a)=x_{a}$.

Assume now that $X_{a}$ contains at least two points. In this case we can effectively pick an element in $X_{a}$ ! Indeed, there is a maximal sequence $u \in 2^{<\alpha}$ such that $u \subseteq x$ for each $x \in X_{a}$. Then the set $X_{a}^{\text {left }}=\{x \in X$ : $\left.u^{\wedge} 0 \subseteq x\right\}$ contains a $\leq_{\text {lex }}$-largest element, which we denote by $\psi(a)$.

To conclude, $\psi$ is a Borel reduction of $\mathrm{E}_{0}$ to the equality on $2^{\alpha}$, i.e. $a \mathrm{E}_{0} b$ iff $\psi(a)=\psi(b)$, which is impossible because $\mathrm{E}_{0}$ is not a smooth Borel equivalence relation (see [1]).

2. The dichotomy. As usual, it will be assumed that the p.q.-o. $\preccurlyeq$ of Theorem 3 is a $\Delta_{1}^{1}$ relation. Let $\approx$ denote the associated equivalence.

Following [2] let, for $\alpha<\omega_{1}^{\mathrm{CK}}, \mathcal{F}_{\alpha}$ be the family of all h.o.p. $\Delta_{1}^{1}$ functions $f:\langle\mathcal{N} ; \preccurlyeq\rangle \rightarrow\left\langle 2^{\alpha} ; \leq_{\text {lex }}\right\rangle$. Then $\mathcal{F}=\bigcup_{\alpha<\omega_{1}^{\mathrm{CK}}} \mathcal{F}_{\alpha}$ is a (countable) $\Pi_{1}^{1}$ set, in a suitable coding system for functions of this type. (See [2] for details.)

Define, for $x, y \in \mathcal{N}, x \equiv y$ iff $f(x)=f(y)$ for any $f \in \mathcal{F}$.

LEMMA 4 (see [2]). $\equiv$ is a $\Sigma_{1}^{1}$ equivalence relation including $\approx$.

Proof. As $\preccurlyeq$ is $\Delta_{1}^{1}$, one gets by a rather standard argument a $\Pi_{1}^{1}$ set $N \subseteq \omega$ and a function $f_{n} \in \mathcal{F}$ for any $n \in N$ so that $\mathcal{F}=\left\{f_{n}: n \in N\right\}$ and the relations $n \in N \wedge f_{n}(x) \leq_{\text {lex }} f_{n}(y)$ and $n \in N \wedge f_{n}(x)<_{\text {lex }} f_{n}(y)$ are presentable in the form $n \in N \wedge \mathcal{O}(x, y)$ and $n \in N \wedge \mathcal{O}^{\prime}(x, y)$ where $\mathcal{O}$, $\mathcal{O}^{\prime}$ are $\Sigma_{1}^{1}$ relations. Now $x \equiv y$ iff $\forall n\left(n \in N \Rightarrow f_{n}(x)=f_{n}(y)\right)$, as required.

CASE 1 : $\equiv$ coincides with $\approx$. Let us show how this implies (I) of Theorem 3 . The set

$$
P=\left\{\langle x, y, n\rangle: x \not \approx y \wedge f_{n}(x) \neq f_{n}(y)\right\}
$$

is $\Pi_{1}^{1}$ and, by the assumption of Case 1, its projection on $x, y$ coincides with the complement of $\approx$. Let $Q \subseteq P$ be a $\Pi_{1}^{1}$ set uniformizing $P$ in the sense of $\mathcal{N}^{2} \times \omega$. Then $Q$ is $\Delta_{1}^{1}$ because

$$
Q(x, y, n) \Leftrightarrow x \not \approx y \wedge \forall n^{\prime} \neq n\left(\neg Q\left(x, y, n^{\prime}\right)\right) .
$$

It follows that $N^{\prime}=\{n: \exists x, y Q(x, y, n)\} \subseteq N$ is $\Sigma_{1}^{1}$. Therefore by the $\Sigma_{1}^{1}$ separation theorem there is a $\Delta_{1}^{1}$ set $M$ such that $N^{\prime} \subseteq M \subseteq N\left({ }^{6}\right)$.

Consider a $\Delta_{1}^{1}$ enumeration $M=\left\{n_{l}: l \in \omega\right\}$. For any $l, f_{n_{l}} \in$ $\mathcal{F}_{\alpha}$ for some ordinal $\alpha=\alpha_{l}<\omega_{1}^{\mathrm{CK}}$. Another standard argument (see

$\left({ }^{6}\right)$ Harrington et al. [2] use a general reflection theorem to get such a set, but a more elementary reasoning sometimes has advantage. 
[2]) shows that in this case (e.g. when $M \subseteq N$ is a $\Delta_{1}^{1}$ set) the ordinals $\alpha_{l}$ are bounded by some $\alpha<\omega_{1}^{\mathrm{CK}}$. It follows that the function $h(x)=$ $f_{n_{0}}(x)^{\wedge} f_{n_{1}}(x)^{\wedge} f_{n_{2}}(x)^{\wedge} \ldots \wedge f_{n_{l}}(x)^{\wedge} \ldots$ belongs to some $\mathcal{F}_{\beta}, \beta \leq \alpha \cdot \omega$. On the other hand, by the construction we have $x \approx y \Leftrightarrow h(x)=h(y)$, hence $h$ satisfies (I) of Theorem 3 .

CASE $2: \approx \varsubsetneqq \equiv$. Assuming this we work towards (II) of Theorem 3 .

3. The domain of singularity. By the assumption the $\Sigma_{1}^{1}$ set $A=$ $\{x: \exists y(x \approx y \wedge x \not \equiv y)\}$ is non-empty.

Define $X \equiv Y$ iff we have $\forall x \in X \exists y \in Y(x \equiv y)$ and vice versa.

Proposition 5. Let $X, Y \subseteq A$ be non-empty $\Sigma_{1}^{1}$ sets satisfying $X \equiv Y$. Then the sets

$$
\begin{aligned}
& P_{+}=\{\langle x, y\rangle \in X \times Y: x \equiv y \wedge x \preccurlyeq y\}, \quad \text { and } \\
& P_{-}=\{\langle x, y\rangle \in X \times Y: x \equiv y \wedge x \preccurlyeq y\}
\end{aligned}
$$

are non-empty $\Sigma_{1}^{1}$ sets, their projections $\left({ }^{7}\right) \mathrm{pr}_{1} P^{+}$and $\mathrm{pr}_{1} P^{-}$are $\Sigma_{1}^{1}$ dense in $X\left({ }^{8}\right)$, while the projections $\mathrm{pr}_{2} P^{+}$and $\mathrm{pr}_{2} P^{-}$are $\Sigma_{1}^{1}$-dense in $Y$.

Proof. The density easily follows from the non-emptiness, so let us concentrate on the latter. We prove that $P_{+} \neq \emptyset$.

Suppose on the contrary that $P_{+}=\emptyset$. Then there is a single function $f \in \mathcal{F}$ such that the set $\{\langle x, y\rangle \in X \times Y: f(x)=f(y) \wedge x \preccurlyeq y\}$ is empty. (See the reasoning in Case 1 of Section 2.) Define

$$
X_{\infty}=\{x: \forall y \in Y(f(x)=f(y) \Rightarrow x \npreceq y)\},
$$

so that $X_{\infty}$ is a $\Pi_{1}^{1}$ set and $X \subseteq X_{\infty}$ but $Y \cap X_{\infty}=\emptyset$. Using separation, we can easily define an increasing sequence of sets

$$
X=X_{0} \subseteq U_{0} \subseteq X_{1} \subseteq U_{1} \subseteq \ldots \subseteq X_{n} \subseteq U_{n} \subseteq \ldots \subseteq X_{\infty}
$$

so that $U_{n}=\left\{x^{\prime}: \exists x \in X_{n}\left(f(x)=f\left(x^{\prime}\right) \wedge x \preccurlyeq x^{\prime}\right)\right\}$ while $X_{n+1} \in \Delta_{1}^{1}$ for all $n$. (Note that if $X_{n} \subseteq X_{\infty}$ and $U_{n}$ is defined as indicated then $U_{n} \subseteq X_{\infty}$ too.) Moreover, a proper execution of the construction $\left({ }^{9}\right)$ allows getting the final set $U=\bigcup_{n} U_{n}=\bigcup_{n} X_{n}$ in $\Delta_{1}^{1}$. Note that $X \subseteq U$, but $Y \cap U=\emptyset$ since $U \subseteq X_{\infty}$.

Put $f^{\prime}(x)=f(x)^{\wedge} 1$ whenever $x \in U$, and $f^{\prime}(x)=f(x)^{\wedge} 0$ otherwise. We assert that $f^{\prime} \in \mathcal{F}$. Indeed, suppose that $x^{\prime} \preccurlyeq y^{\prime}$; we prove $f^{\prime}\left(x^{\prime}\right) \leq_{\text {lex }} f^{\prime}\left(y^{\prime}\right)$.

$\left({ }^{7}\right)$ For a set $P \subseteq \mathcal{N}^{2}, \mathrm{pr}_{1} P$ and $\mathrm{pr}_{2} P$ have the obvious meaning of the projections on the resp. 1st and 2 nd copy of $\mathcal{N}$.

$\left({ }^{8}\right)$ That is, each of them intersects any non-empty $\Sigma_{1}^{1}$ set $X^{\prime} \subseteq X$.

$\left({ }^{9}\right)$ We refer to the proof of an "invariant" effective separation theorem in [1], which includes a similar construction. 
It can be assumed that $f\left(x^{\prime}\right)=f\left(y^{\prime}\right)$. It remains to check that $x^{\prime} \in U \Rightarrow$ $y^{\prime} \in U$, which easily follows from the definition of the sets $U_{n}$. Thus $f^{\prime} \in \mathcal{F}$.

However, clearly $f^{\prime}(x) \neq f^{\prime}(y)$, hence $x \not \equiv y$, whenever $x \in X$ and $y \in Y$, which contradicts the assumption that $X \equiv Y$.

Now we prove that $P_{-} \neq \emptyset$. Consider first the case $X=Y$. Suppose on the contrary that $P_{-}=\emptyset$. Then, as above, there is a single function $f \in \mathcal{F}$ such that the set $\left\{\langle x, y\rangle \in X^{2}: f(x)=f(y) \wedge x \npreceq y\right\}$ is empty, so that $\equiv$ and $\approx$ coincide on $X$. Our plan is to find functions $f^{\prime}, f^{\prime \prime} \in \mathcal{F}$ such that

$$
\begin{aligned}
Q^{\prime} & =\left\{\langle x, y\rangle \in X \times \mathcal{N}: f^{\prime}(x)=f^{\prime}(y) \wedge y \npreceq x\right\}, \\
Q^{\prime \prime} & =\left\{\langle x, y\rangle \in X \times \mathcal{N}: f^{\prime \prime}(x)=f^{\prime \prime}(y) \wedge x \npreceq y\right\}
\end{aligned}
$$

are empty sets; then $Q=\{\langle x, y\rangle \in X \times \mathcal{N}: x \equiv y \wedge y \not \approx x\}=\emptyset$, which contradicts $\emptyset \neq X \subseteq A$.

Let us find $f^{\prime}$; the case of the other function is similar. Define

$$
X_{\infty}=\left\{x: \forall x^{\prime} \in X\left(f(x)=f\left(x^{\prime}\right) \Rightarrow x \preccurlyeq x^{\prime}\right)\right\},
$$

so that $X_{\infty}$ is $\Pi_{1}^{1}$ and $X \subseteq X_{\infty}$. As above there is a sequence of sets

$$
X=X_{0} \subseteq U_{0} \subseteq X_{1} \subseteq U_{1} \subseteq \ldots \subseteq X_{n} \subseteq U_{n} \subseteq \ldots \subseteq X_{\infty}
$$

such that $U_{n}=\left\{u: \exists x \in X_{n}(f(x)=f(u) \wedge u \preccurlyeq x)\right\}$ while $X_{n+1} \in \Delta_{1}^{1}$ for all $n$ and the final set $U=\bigcup_{n} U_{n}=\bigcup_{n} X_{n}$ belongs to $\Delta_{1}^{1}$.

Set $f^{\prime}(x)=f(x)^{\wedge} 0$ whenever $x \in U$, and $f^{\prime}(x)=f(x)^{\wedge} 1$ otherwise. Then $f^{\prime} \in \mathcal{F}$. We prove that $f^{\prime}$ witnesses that $Q^{\prime}=\emptyset$. Consider any $x \in X$ and $y \in \mathcal{N}$ such that $f^{\prime}(x)=f^{\prime}(y)$. Then in particular $f(x)=f(y)$ and $x \in U \Leftrightarrow y \in U$, so that $y \in U$ because we know that $x \in X \subseteq U$. Thus $y \in X_{\infty}$, so by definition $y \preccurlyeq x$, as required.

Finally, we prove $P_{-} \neq \emptyset$ in the general case. By the result for the case $X=Y$, the $\Sigma_{1}^{1}$ set $P^{\prime}=\left\{\left\langle x, x^{\prime}\right\rangle \in X^{2}: x \equiv x^{\prime} \wedge x \npreceq x^{\prime}\right\}$ is non-empty. Let $X^{\prime}=\left\{x^{\prime} \in X: \exists x P^{\prime}\left(x, x^{\prime}\right)\right\}$ and $Y^{\prime}=\left\{y \in Y: \exists x^{\prime} \in X^{\prime}\left(x^{\prime} \equiv y\right)\right\}$, so that $X^{\prime}, Y^{\prime}$ are $\Sigma_{1}^{1}$ sets satisfying $X^{\prime} \equiv Y^{\prime}$. By the result for $P_{+}$there exist $x^{\prime} \in X^{\prime}$ and $y \in Y^{\prime}$ satisfying $x^{\prime} \equiv y$ and $y \preccurlyeq x^{\prime}$. Now there is $x \in X$ such that $x \equiv x^{\prime}$ and $x \npreceq x^{\prime}$. Then $x \equiv y$ and $x \npreceq y$, as required.

4. The forcing notions involved. Our further strategy will be the following. We shall define a generic extension of the universe $\mathbf{V}$ (where Theorem 3 is being proved) in which there exists a function $F$ which witnesses (II) of Theorem 3. However, as the existence of such a function is a $\Sigma_{2}^{1}$ statement, we obtain the result for $\mathbf{V}$ by the Shoenfield absoluteness theorem $\left({ }^{10}\right)$.

Definition 6. $\mathbb{P}$ is the collection of all non-empty $\Sigma_{1}^{1}$ sets $X \subseteq A$.

$\left({ }^{10}\right)$ In fact, the proof can be conducted without any use of metamathematics, as in $[1]$, but at the cost of longer reasoning. 
It is a standard fact that $\mathbb{P}$ (the Gandy forcing) forces a real which is the only real which belongs to every set in the generic set $G \subseteq \mathbb{P}$. (We identify $\Sigma_{1}^{1}$ sets in the ground universe $\mathbf{V}$ with their copies in the extension.)

Definition 7. $\mathbb{P}_{2}^{+}$is the collection of all non-empty $\Sigma_{1}^{1}$ sets $P \subseteq A^{2}$ such that $P(x, y) \Rightarrow x \equiv y \wedge x \preccurlyeq y$. The collection $\mathbb{P}_{2}^{-}$is defined similarly but with the requirement $P(x, y) \Rightarrow x \equiv y \wedge x \npreceq y$ instead.

Both $\mathbb{P}_{2}^{+}$and $\mathbb{P}_{2}^{-}$are non-empty forcing notions by Proposition 5 . Each of them forces a pair of reals $\langle x, y\rangle \in A^{2}$ satisfying resp. $x \preccurlyeq y$ and $x \nprec y$.

DeFinition 8. $\mathbb{P}_{\equiv}^{2}$ is the collection of all sets of the form $\Upsilon=X \times Y$ where $X, Y$ are sets in $\mathbb{P}$ satisfying $X \equiv Y$.

LEMma 9. $\mathbb{P}_{\equiv}^{2}$ forces a pair of reals $\langle x, y\rangle$ such that $x \npreceq y$.

Proof. Suppose that, on the contrary, a condition $\Upsilon_{0}=X_{0} \times Y_{0}$ in $\mathbb{P}_{\equiv}^{2}$ forces $x \preccurlyeq y$. Consider a more complicated forcing $\mathfrak{P}$ which consists of forcing conditions of the form $\mathfrak{p}=\left\langle\Upsilon, P, \Upsilon^{\prime}, Q\right\rangle$, where $\Upsilon=X \times Y$ and $\Upsilon^{\prime}=X^{\prime} \times Y^{\prime}$ belong to $\mathbb{P}_{\equiv}^{2}, P \in \mathbb{P}_{2}^{+}, P \subseteq Y \times X^{\prime}, Q \in \mathbb{P}_{2}^{-}, Q \subseteq X \times Y^{\prime}$, and the sets $\operatorname{pr}_{1} P \subseteq Y, \operatorname{pr}_{2} P \subseteq X^{\prime}, \operatorname{pr}_{1} Q \subseteq X$ and $\operatorname{pr}_{2} Q \subseteq Y^{\prime}$ are $\Sigma_{1}^{1}$-dense in resp. $Y, X^{\prime}, X, Y^{\prime}$.

For instance, setting $P_{0}=\left\{\left\langle y, x^{\prime}\right\rangle \in Y_{0} \times X_{0}: y \equiv x^{\prime} \wedge y \preccurlyeq x^{\prime}\right\}$ and $Q_{0}=\left\{\left\langle x, y^{\prime}\right\rangle \in X_{0} \times Y_{0}: x \equiv y^{\prime} \wedge x \npreceq y^{\prime}\right\}$, we get a condition $\mathfrak{p}_{0}=$ $\left\langle\Upsilon_{0}, P_{0}, \Upsilon_{0}, Q_{0}\right\rangle \in \mathfrak{P}$ by Proposition 5 .

It is the principal fact that if $\mathfrak{p}=\left\langle\Upsilon, P, \Upsilon^{\prime}, Q\right\rangle \in \mathfrak{P}$ and we strengthen one of the components within the corresponding forcing notion then this can be appropriately reflected in the other components. To be concrete assume that, for instance, $P^{*} \in \mathbb{P}_{2}^{+}, P^{*} \subseteq P$, and find a condition $\mathfrak{p}_{1}=\left\langle\Upsilon_{1}, P_{1}, \Upsilon_{1}^{\prime}, Q_{1}\right\rangle \in$ $\mathfrak{P}$ satisfying $\Upsilon_{1} \subseteq \Upsilon, \Upsilon_{1}^{\prime} \subseteq \Upsilon^{\prime}, P_{1} \subseteq P^{*}$, and $Q_{1} \subseteq Q$.

Assume that $\Upsilon=X \times Y$ and $\Upsilon^{\prime}=X^{\prime} \times Y^{\prime}$. Consider the non-empty $\Sigma_{1}^{1}$ sets $Y_{2}=\operatorname{pr}_{1} P^{*} \subseteq Y$ and $X_{2}=\left\{x \in X: \exists y \in Y_{2}(x \equiv y)\right\}$. It follows from Proposition 5 that $Q_{1}=\left\{\langle x, y\rangle \in Q: x \in X_{2}\right\} \neq \emptyset$, hence $Q_{1}$ is a condition in $\mathbb{P}_{2}^{-}$and $X_{1}=\operatorname{pr}_{1} Q_{1}$ is a non-empty $\Sigma_{1}^{1}$ subset of $X_{2} \subseteq X$.

The set $Y_{1}=\left\{y \in Y_{2}: \exists x \in X_{1}(x \equiv y)\right\}$ satisfies $X_{1} \equiv Y_{1}$, therefore $\Upsilon_{1}=X_{1} \times Y_{1} \in \mathbb{P}_{\equiv}^{2}$. Furthermore, $P_{1}=\left\{\langle y, x\rangle \in P^{*}: y \in Y_{1}\right\} \in \mathbb{P}_{2}^{+}$.

Put $X_{1}^{\prime}=\operatorname{pr}_{2} P_{1} \subseteq X^{\prime}$ and $Y_{1}^{\prime}=\operatorname{pr}_{2} Q_{1} \subseteq Y^{\prime}$. Notice that $Y_{1} \equiv X_{1}^{\prime}$ because any condition in $\mathbb{P}_{2}^{+}$is a subset of $\equiv$, similarly $X_{1} \equiv Y_{1}^{\prime}$, and $X_{1} \equiv Y_{1}$ (see above). It follows that $X_{1}^{\prime} \equiv Y_{1}^{\prime}$, hence $\Upsilon_{1}^{\prime}=X_{1}^{\prime} \times Y_{1}^{\prime}$ is a condition in $\mathbb{P}_{\equiv}^{2}$.

Now $\mathfrak{p}_{1}=\left\langle\Upsilon_{1}, P_{1}, \Upsilon_{1}^{\prime}, Q_{1}\right\rangle \in \mathfrak{P}$ as required.

We conclude that $\mathfrak{P}$ forces "quadruples" of reals $\left\langle x, y, x^{\prime}, y^{\prime}\right\rangle$ such that the pairs $\langle x, y\rangle$ and $\left\langle x^{\prime}, y^{\prime}\right\rangle$ are $\mathbb{P}_{\equiv}^{2}$-generic, hence satisfy $x \preccurlyeq y$ and $x^{\prime} \preccurlyeq y^{\prime}$ provided the generic set contains $\Upsilon_{0}$ - by the assumption above. Furthermore, the pair $\left\langle y, x^{\prime}\right\rangle$ is $\mathbb{P}_{2}^{+}$-generic, hence $y \preccurlyeq x^{\prime}$, while the pair $\left\langle x, y^{\prime}\right\rangle$ is $\mathbb{P}_{2}^{-}$-generic, hence $x \npreceq y^{\prime}$, which is a contradiction. 
5. The splitting construction. Let, in the universe $\mathbf{V}, \kappa=2^{\aleph_{0}}$. Let $\mathbf{V}^{+}$be a $\kappa$-collapse extension of $\mathbf{V}$.

Our aim is to define, in $\mathbf{V}^{+}$, a splitting system of sets which leads to a function $F$ satisfying (II) of Theorem 3. Let us fix two points before the construction starts.

First, as the forcing notions involved are countable in $\mathbf{V}$, there exist, in $\mathbf{V}^{+}$, enumerations $\{D(n): n \in \omega\},\left\{D_{2}(n): n \in \omega\right\}$, and $\left\{D^{2}(n): n \in \omega\right\}$ of all open dense sets in resp. $\mathbb{P}, \mathbb{P}_{2}^{+}, \mathbb{P}_{\equiv}^{2}$, which (the dense sets) belong to $\mathbf{V}$, such that $D(n+1) \subseteq D(n)$ etc. for each $n$.

Second, we introduce the notion of a crucial pair. A pair $\langle u, v\rangle$ of binary sequences $u, v \in 2^{n}$ is called crucial iff $u=1^{k \wedge} 0^{\wedge} w$ and $v=0^{k \wedge} 1^{\wedge} w$ for some $k<n$ and $w \in 2^{n-k-1}$. One easily sees that the graph of all crucial pairs in $2^{n}$ is actually a chain connecting all members of $2^{n}$.

We define, in $\mathbf{V}^{+}$, a system of sets $X_{u} \in \mathbb{P}$, where $u \in 2^{<\omega}$, and sets $P_{u v} \in \mathbb{P}_{2}^{+},\langle u, v\rangle$ being a crucial pair in some $2^{n}$, satisfying the following conditions:

(1) $X_{u} \in D(n)$ whenever $u \in 2^{n} ; X_{u \wedge i} \subseteq X_{u}$;

(2) if $\langle u, v\rangle$ is a crucial pair in $2^{n}$ then $P_{u v} \in D_{2}(n)$ and $P_{u^{\wedge} i, v^{\wedge} i} \subseteq P_{u v}$;

(3) if $u, v \in 2^{n}$ and $u(n-1) \neq v(n-1)$ then $X_{u} \times X_{v} \in \mathbb{P}_{\equiv}^{2}, X_{u} \times X_{v} \in$ $D^{2}(n)$, and $X_{u} \cap X_{v}=\emptyset$;

(4) if $\langle u, v\rangle$ is a crucial pair in $2^{n}$ then $\operatorname{pr}_{1} P_{u v}=X_{u}$ and $\operatorname{pr}_{2} P_{u v}=X_{v}$.

Why does this imply the existence of a required function? First of all for any $a \in 2^{\omega}$ (in $\mathbf{V}^{+}$) the sequence of sets $X_{a \mid n}$ is $\mathbb{P}$-generic over $\mathbf{V}$ by (1), therefore the intersection $\bigcap_{n \in \omega} X_{a \uparrow n}$ is a singleton. Let $F(a) \in \mathcal{N}$ be its only element.

It does not take much effort to prove that $F$ is continuous and 1-1.

Consider $a, b \in 2^{\omega}$ satisfying $a \mathbb{Z}_{0} b$. Then $a(n) \neq b(n)$ for infinitely many $n$, hence the pair $\langle F(a), F(b)\rangle$ is $\mathbb{P}_{\equiv}^{2}$-generic by (3), thus $F(a)$ and $F(b)$ are $\preccurlyeq$-incomparable by Lemma 9 .

Consider $a, b \in 2^{\omega}$ satisfying $a \leq_{0} b$. We may assume that $a$ and $b$ are $\leq_{0}$-neighbours, i.e. $a=1^{k \wedge} 0^{\wedge} c$ while $b=0^{k \wedge} 1^{\wedge} c$ for some $k \in \omega$ and $c \in 2^{\omega}$. Then by (2) the sequence of sets $P_{a \uparrow n, b \uparrow n}, n>k$, is $\mathbb{P}_{2}^{+}$-generic, hence it results in a pair of reals satisfying $x \preccurlyeq y$. However, $x=F(a)$ and $y=F(b)$ by $(4)$.

The construction of a splitting system. We argue in $\mathbf{V}^{+}$.

Suppose that the construction has been completed up to a level $n$; we will expand it to the next level. From now on $s, t$ will denote sequences in $2^{n}$ while $u, v$ will denote sequences in $2^{n+1}$.

To start with, we set $X_{s^{\wedge} i}=X_{s}$ for all $s \in 2^{n}$ and $i=0,1$, and $P_{s^{\wedge} i, t^{\wedge} i}=$ $P_{s t}$ whenever $i=0,1$ and $\langle s, t\rangle$ is a crucial pair in $2^{n}$. 
For the "initial" crucial pair $\left\langle 1^{n \wedge} 0,0^{n \wedge} 1\right\rangle$ at this level let $P_{1^{n \wedge} 0,0^{n \wedge}}=$ $X_{1^{n \wedge}} \times X_{0^{n \wedge}}=X_{1^{n}} \times X_{0^{n}}$. Then $\left.P_{1^{n \wedge} 0,0^{n \wedge 1}} \in \mathbb{P}^{2} \equiv{ }^{11}\right)$.

This ends the definition of "initial values" at the $(n+1)$ th level. The plan is to gradually "shrink" the sets in order to fulfill the requirements.

STEP 1. We take care of item (1). Consider an arbitrary $u_{0}=s_{0} \wedge i \in$ $2^{n+1}$. As $D(n)$ is dense there is a set $X^{\prime} \in D(n)$ with $X^{\prime} \subseteq X_{u_{0}}$. The intention is to take $X^{\prime}$ as the "new" $X_{u_{0}}$. But this change has to be expanded through the chain of crucial pairs, in order to preserve (4).

Thus put $X_{u_{0}}^{\prime}=X^{\prime}$. Suppose that $X_{u}^{\prime}$ has been defined and is included in $X_{u}$, the "old" version, for some $u \in 2^{n+1}$, and $\langle u, v\rangle$ is a crucial pair, $v \in 2^{n+1}$ being not yet encountered. Define $P_{u v}^{\prime}=\left(X_{u}^{\prime} \times \mathcal{N}\right) \cap P_{u v}$ and $X_{v}^{\prime}=\operatorname{pr}_{2} P_{u v}^{\prime}$. Clearly (4) holds for the "new" sets $X_{u}^{\prime}, X_{v}^{\prime}$, and $P_{u v}^{\prime}$.

The construction describes how the original change from $X_{u_{0}}$ to $X_{u_{0}}^{\prime}$ spreads through the chain of crucial pairs in $2^{n+1}$, resulting in a system of new sets, $X_{u}^{\prime}$ and $P_{u v}^{\prime}$, which satisfy (1) for the particular $u_{0} \in 2^{n+1}$. We iterate this construction consecutively for all $u_{0} \in 2^{n+1}$, getting finally a system of sets satisfying (1) (fully) and (4), which we shall denote by $X_{u}$ and $P_{u v}$ from now on.

SteP 2. We take care of item (3). Fix a pair of $u_{0}$ and $v_{0}$ in $2^{n+1}$ such that $u_{0}(n)=0$ and $v_{0}(n)=1$. By the density of $D^{2}(n)$, there is a set $X_{u_{0}}^{\prime} \times X_{v_{0}}^{\prime} \in D^{2}(n)$ included in $X_{u_{0}} \times X_{v_{0}}$. We may assume that $X_{u_{0}}^{\prime} \cap X_{v_{0}}^{\prime}=\emptyset$. (Indeed, it easily follows from Proposition 5, for $P_{-}$, that there exist reals $x_{0} \in X_{u_{0}}$ and $y_{0} \in X_{v_{0}}$ satisfying $x_{0} \equiv y_{0}$ but $x_{0} \neq y_{0}$, say $x_{0}(k)=0$ while $y_{0}(k)=1$. Define

$$
X=\left\{x \in X_{0}: x(k)=0 \wedge \exists y \in Y_{0}(y(k)=1 \wedge x \equiv y)\right\},
$$

and $Y$ correspondingly; then $X \equiv Y$ and $X \cap Y=\emptyset$.)

Spread the change from $X_{u_{0}}$ to $X_{u_{0}}^{\prime}$ and from $X_{v_{0}}$ to $X_{v_{0}}^{\prime}$ through the chain of crucial pairs in $2^{n+1}$, by the method of Step 1 , until the wave of spreading from $u_{0}$ meets the wave of spreading from $u_{0}$ at the "meeting" crucial pair $\left\langle 1^{n \wedge} 0,0^{n \wedge} 1\right\rangle$. This leads to a system of sets $X_{u}^{\prime}$ and $P_{u v}^{\prime}$ which satisfy (3) for the particular pair $\left\langle u_{0}, v_{0}\right\rangle$ and still satisfy (4) possibly except for the "meeting" crucial pair $\left\langle 1^{n \wedge} 0,0^{n \wedge} 1\right\rangle$ (for which basically the set $P_{1^{n \wedge} 0,0^{n \wedge} 1}^{\prime}$ is not yet defined at this step).

Note that Step 1 leaves $P_{1^{n} \wedge} 0,0^{n \wedge} 1$ in the form $X_{1^{n} \wedge} \times X_{0^{n \wedge}}$ (where $X_{1^{n \wedge} 0}$ and $X_{0^{n \wedge}}$ are the "versions" at the end of Step 1). We now have the "new" sets, $X_{1^{n \wedge}}^{\prime}$ and $X_{0^{n \wedge} 1}^{\prime}$, included in resp. $X_{1^{n \wedge}}$ and $X_{0^{n \wedge}}$ and satisfying $X_{0^{n} \wedge}^{\prime} \equiv X_{0^{n \wedge}}^{\prime}$ (because we had $X_{u_{0}}^{\prime} \equiv X_{v_{0}}^{\prime}$ at the beginning of

$\left({ }^{11}\right)$ It easily follows from (2) and (4) that $X_{s} \equiv X_{t}$ for all $s, t \in 2^{n}$, because $s$ and $t$ are connected in $2^{n}$ by a unique chain of crucial pairs. 
the change). It remains to define $P_{1^{n} \wedge_{0,0^{n} \wedge}}^{\prime}=X_{1^{n} \wedge_{0}}^{\prime} \times X_{0^{n} \wedge_{1}}^{\prime}$. This ends the consideration of the pair $\left\langle u_{0}, v_{0}\right\rangle$.

Applying this construction consecutively for all pairs of $u_{0} \in P_{0}$ and $v_{0} \in P_{1}$ (including the pair $\left\langle 1^{n \wedge} 0,0^{n \wedge} 1\right\rangle$ ) we finally get a system of sets satisfying (1), (3), and (4), which will be denoted still by $X_{u}$ and $P_{u v}$.

STEP 3. We finally take care of (2). Consider a crucial pair $\left\langle u_{0}, v_{0}\right\rangle$ in $2^{n+1}$. By density, there exists a set $P_{u_{0}, v_{0}}^{\prime} \in D_{2}(n)$ with $P_{u_{0}, v_{0}}^{\prime} \subseteq P_{u_{0}, v_{0}}$. (In the case when $\left\langle u_{0}, v_{0}\right\rangle$ is the pair $\left\langle 1^{n \wedge} 0,0^{n \wedge} 1\right\rangle$ we rather apply Proposition 5 to obtain the set $P_{u_{0}, v_{0}}^{\prime}$.)

Define $X_{u_{0}}^{\prime}=\operatorname{pr}_{1} P_{u_{0}, v_{0}}^{\prime}$ and $X_{v_{0}}^{\prime}=\operatorname{pr}_{2} P_{u_{0}, v_{0}}^{\prime}$ and spread this change through the chain of crucial pairs in $2^{n+1}$. (Note that $X_{u_{0}}^{\prime} \equiv X_{v_{0}}^{\prime}$ as sets in $\mathbb{P}_{\equiv}^{2}$ are included in $\equiv$. This keeps $X_{u}^{\prime} \equiv X_{v}^{\prime}$ for all $u, v \in 2^{n+1}$ through the spreading.)

Executing this step for all crucial pairs in $2^{n+1}$, we finally end the construction, in $\mathbf{V}^{+}$, of a system of sets satisfying (1) through (4).

\section{References}

[1] L. A. Harrington, A. S. Kechris and A. Louveau, A Glimm-Effros dichotomy for Borel equivalence relations, J. Amer. Math. Soc. 3 (1990), 903-928.

[2] L. A. Harrington, D. Marker and S. Shelah, Borel orderings, Trans. Amer. Math. Soc. 310 (1988), 293-302.

Department of Mathematics

Moscow Transport Engineering Institute

Obraztsova 15

Moscow 101475, Russia

E-mail: kanovei@mech.math.msu.su and kanovei@math.uni-wuppertal.de 